\title{
Recurrent Idiopathic Gingival Hyperplasia
}

\author{
Dr. Junima Rajkarnikar, ${ }^{1}$ Dr. Bikash Veer Shrestha, ${ }^{2}$ Dr. Santhosh Kumar ${ }^{3}$ \\ ${ }^{1}$ Department of Periodontology, Nepal Medical College, Kathmandu, Nepal; \\ ${ }^{2}$ Department of Orthodontics, Nepal Medical College, Kathmandu, Nepal; \\ ${ }^{3}$ Department of Periodontology, Manipal College of Dental Sciences, Manipal Acadamy of Higher Education, \\ Manipal, Karnataka, India.
}

\begin{abstract}
Increase in size of the gingiva is termed as gingival enlargement. Most common type of gingival enlargement is inflammatory, which his caused due to plaque accumulation and improper oral hygiene maintenance. Orthodontic therapy can often lead to failure to improve oral hygiene. This case describes a recurrent, progressive gingival enlargement of a 19 year old female orthodontic patient in which gingivectomy was performed and repeated, which subsequently failed. Hence modified Widman's flap was performed with medical supplements. Periodic periodontal check up is required in orthodontic cases to control the gingival inflammation. Patient compliance is also very important in such cases. There should be proper co-operation between the Orthodontist and Periodontist for successful treatment of gingival hyperplasia. Patients with such conditions should be carefully monitored and checked to avoid the recurrence and avoid further progression into chronic periodontitis.

Keywords: Gingival enlargement; hyperplasia; orthodontic patient; recurrent.
\end{abstract}

\section{INTRODUCTION}

Gingival enlargement or overgrowth is a clinical descriptive term for increases in size of gingiva. ${ }^{1}$ Most commonly, gingival enlargement can be due to plaque accumulation, poor oral hygiene, inadequate nutrition, or systemic hormonal stimulation. $^{2}$ Gingival enlargements are also seen in blood dyscrasias like leukaemia and thrombocytopenia. ${ }^{1}$ Idiopathic gingival enlargement is a rare type of gingival enlargement that has no definite cause. Inability to properly clean the teeth surfaces clean and presence of plaque is considered as one of the main factors responsible for the development of gingivitis as orthodontic brackets and elastics might interfere with effective removal of plaque. ${ }^{3}$ There is also shift in the composition of bacteria due to orthodontic treatment as it can increase the bacterial retention. ${ }^{4}$ Gingival enlargement in orthodontic patient can lead to pseudo pocket, where the hyperplasia can lead to deep artificial pockets and this has been related to shift to more anaerobic flora like B. intermedius, Spirochetes, B. forsythus, T. denticola, P. nigrescens, etc. ${ }^{5}$ This is a case report of an unusual case of a non-syndromic recurrent idiopathic gingival enlargement.

\section{Correspondence:}

Dr. Junima Rajkarnikar

Department of Periodontics, Nepal Medical College, Kathmandu, Nepal.

email: drjunima@gmail.com

Citation

Rajkarnikar J, Shrestha BV, Kumar S. Recurrent Idiopathic Gingival Hyperplasia. J Nepal Soc Perio Oral Implantol. 2019;3(5):35-7.

\section{CASE REPORT}

A 19 year old female patient from Besigaun, Jorpati was referred to the department of Periodontics, Nepal Medical College Teaching Hospital from the department of Orthodontics of the same for the treatment of gingival enlargement. Her first visit to the Department of Periodontics was in May 2018 during which Phase I therapy was completed with oral prophylaxis and oral hygiene instructions. Chlorhexidine mouthwash $0.2 \%$ (Hexidine) $10 \mathrm{ml}$ BD was given for seven days. She gave the history of gingival enlargement which was evident since few years. Gingival tissue was pale pink, enlarged, firm, fibrotic and mild inflammatory. It was painless and there was no difficulty in speech and mastication. There was no history of drugs intake, no mental or physical disorder and no systemic disease present. Family history was non contributory. So the patient was recalled for gingivectomy after a week.

On her second visit, oral examination revealed fibrotic gingival enlargement involving upper and lower anteriors with grade II gingival enlargement involving the papilla and marginal gingiva (Bokenkamp Index - 1994). There

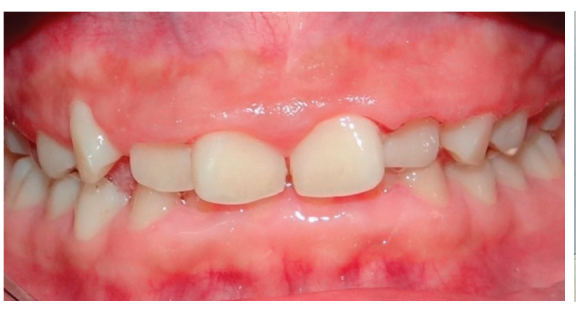

Figure 1: Gingival enlargement prior to orthodontic therapy.

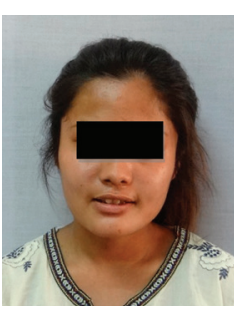

Figure 2: Extraoral view. 


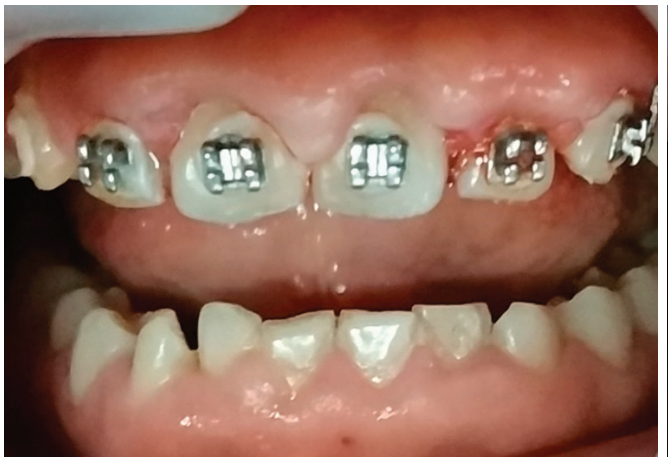

Figure 3: Gingival enlargement after orthodontic therapy.

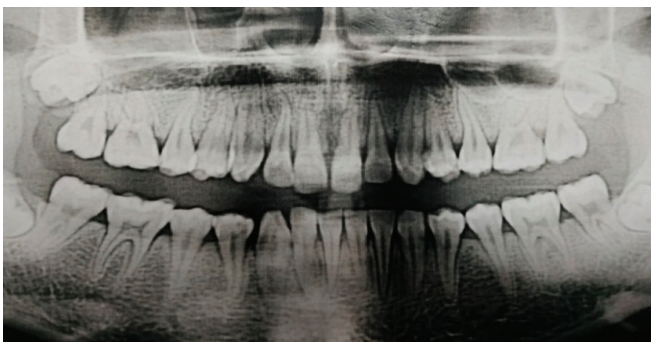

Figure 5: OPG before orthodontic therapy.

were pseudo pockets of 5-6 $\mathrm{mm}$ in the anteriors. There was adequate attached gingiva. No significant alveolar bone loss was evident on orthopantomogram (OPG).

Written informed consent was taken after which gingivectomy procedure was performed under local anaesthesia with respect to upper anteriors with the help of Bard Parker blades and gingivectomy knives. The excised tissue was sent for histopathological examination in $10 \%$ formalin solution. The surgical area was covered with periodontal pack and the patient was recalled after a week.

Histopathological examination revealed hyperparakeratinized stratified squamous epithelium with increase in the connective tissue. Densely arranged collagenfiber bundles with abundance of fibroblasts was reported with mild chronic inflammatory cells. It was diagnosed as inflammatory fibrous gingival hyperplasia.

The second visit of the patient was in June for recurrence of gingival enlargement in the same area. Gingivectomy was

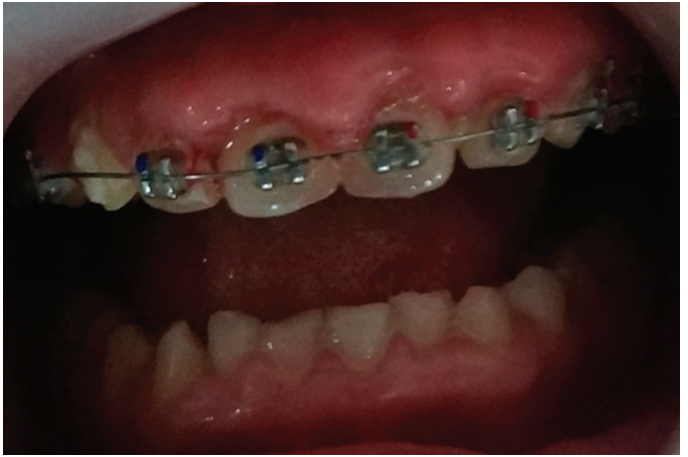

Figure 4: Recurrent gingival enlargement.

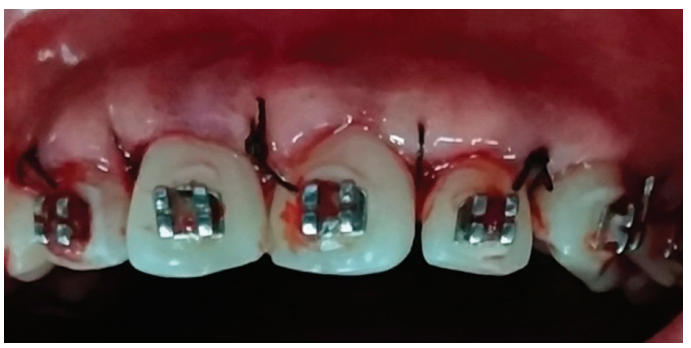

Figure 6: Sutured after modified Widman's flap.

repeated in 15-25 and analgesics were prescribed. Again the patient was referred for the third time from Orthodontics due to recurrence of enlargement during the treatment. Upon clinical examination, bleeding on probing was present with visible plaque. At this point, Orthodontic therapy was advised to be postponed for at least a month. Scaling was performed and oral hygiene reinforced. After a week, modified Widman's flap was performed. Granulation tissue was removed and the area was thoroughly irrigated with normal saline solution after which it was sutured with 4-0 silk (mersilk) sutures. Serratiopeptidase 10mg (Serpa) thrice a day for three days was given to avoid any unnecessary oedema. Folic acid 5mg (Folate) once a day for 15 days along with analgesics was given. Gingivectomy was also performed in lower arch on later date. There was marked improvement in gingival condition, as the enlargement had significantly decreased and it was maintained for 10 months and the patient is undergoing the Orthodontic therapy

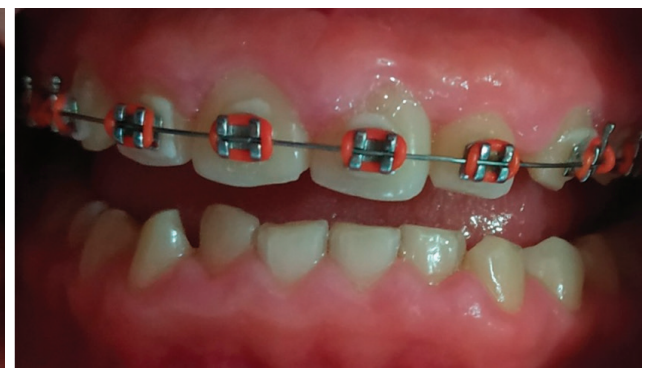

Figure 8: Post operative after six months.

Figure 7: Post-operative after 10 days. 


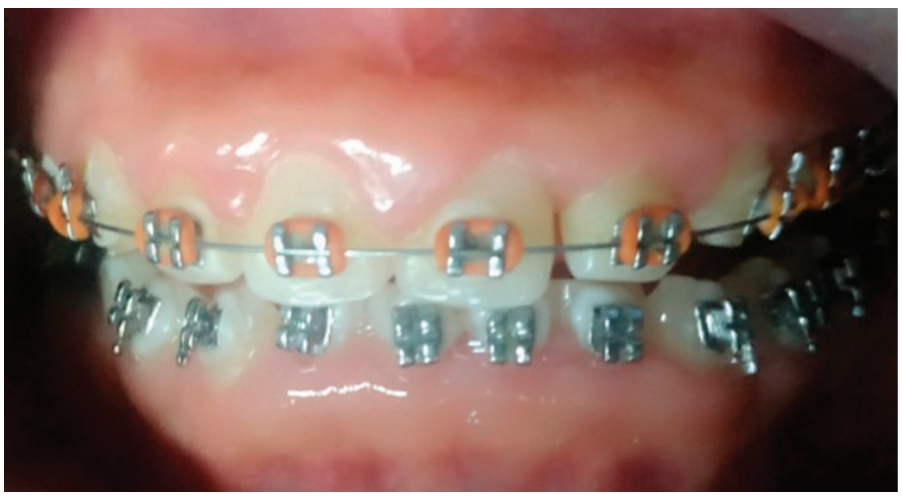

Figure 9: Post-operative after 10 months.

\section{DISCUSSION}

Treatment of gingival enlargement is based on an understanding of the cause of enlargement and the underlying pathologic changes. Non surgical procedures should be adequately performed along with reinforcement of oral hygiene. Gingival enlargements that still persist after that should either be treated by gingivectomy or flap surgery, depending upon the width of the attached gingiva, loss of alveolar bone and number of teeth involved. ${ }^{1}$

We had supplemented our patient with folic acid. Folic acid is required for the activation of collagenase. It binds to plaque-derived endotoxin and prevents stimulation of endotoxin complement immune system. This will decrease local hyperplastic changes. Absence of inflammation seen in folic acid deficiency is the result of deficiency induced granulocytopenia. ${ }^{6}$ A strong interrelationship exists between sex hormones and folate coenzymes. End organs may be affected more severely than other areas. ${ }^{7}$

Hugoson in 1971 said that there is significant correlation between the level of oestrogen and progesterone and the severity of gingival inflammation which is not accompanied by an increase in bacterial plaque. ${ }^{8}$ Gingival hyperplasia in Orthodontic patients can lead to pseudo-pocketing, where there is no attachment loss but shift to more anaerobic flora occurs. This necessitates care on the part of the patient to ensure the avoidance of plaque retention. Also, resin system aligners has been shown to result in a lower plaque index when compared to those with fixed orthodontic appliances. ${ }^{9}$

In a study done in different age group of fixed Orthodontic treatment patient, highest frequency of gingival enlargement was seen among the adolescents (10-19 years). Those who brushed three times a day did not have any enlargement. Those who brushed once a day had highest percentage of grade 2 gingival enlargement. Gingival enlargement was mostly due to lack of patient cooperation and less patient compliance. $^{10}$

Oral hygiene reinforcement is important for Orthodontic patients at each visit. Hormonal changes in puberty is one of the cause of gingival enlargement. Nutritional deficiency also aggravates the condition. Therefore it is important to know the cause of the condition and do proper treatment planning along with periodic maintenance.

\section{REFERENCES}

1. Newman, Takei, Klokkevold, Carranza's Clinical Periodontology. David LC, William VG, Barrie EK, John MN, Jane LF, Philippe PH, Mark BL. 11th ed. Elsevier Saunders 2013;1:84

2. Regezi JA, Sciuba JJ. Connective tissue lesions. In oral pathology: Clinical pathologic correlations. Philadelphia: W.B. Saunders; 1999;1:179-83.

3. Krishnan V, Ambili R, Davidovitch Z, Murphy NC. Gingiva and Orthodontic Treatment. Sem Ortho. 2007;13:257-71.

4. Van Gastel J, Quirynen M, Teughels W, Carels C. The relationships between malocclusion, fixed orthodontic appliances and periodontal disease: A review of the literature. Aus Ortho J. 2007;23:121-9.

5. Alstad S, Zachrisson BU. Longitudinal study of periodontal condition associated with orthodontic treatment in adolescents. Am J Orthod. 1979;5:133-51.

6. George JP, Shobha R, Lazarus FJ. Folic acid: A positive influence on periodontal tissues during health and disease. Int J Health Allied Sci. 2013;2:145-52.

7. Kronman K, Loseche JF. Direct interaction of estradiol and progesterone with Bacteriodes melaninogenicus . Infect Immun. 1982;35:256-63.

8. Hugoson A. Gingivitis in pregnant women. A longitudinal clinical study. Odontol Revy. 1971;22:65-84.

9. Alstad S, Zachrisson BU. Longitudinal study of periodontal condition associated with orthodontic treatment in adolescents. Am J Orthod. 1979;5:133-51.

10. Kumar PS. Sex and the subgingival microbiome: Do female sex steroids affect periodontal bacteria? Periodontol 2000. $2013 ; 61: 103$ 24. 